\title{
Simulating low dimensional QCD with Lefschetz thimbles
}

\author{
Christian Schmidt* \\ Universität Bielefeld, Fakultät für Physik, D-33615 Bielefeld, Germany \\ E-mail: schmidtephysik. uni-bielefeld.de

\section{Felix Ziesché} \\ Universität Bielefeld, Fakultät für Physik, D-33615 Bielefeld, Germany \\ E-mail: fziesche@physik.uni-bielefeld.de
}

\begin{abstract}
Non-perturbative lattice QCD calculations at non vanishing baryon number density are hampered by the QCD sign problem. The path integral, that in lattice QCD is calculated numerically, becomes highly oscillating. One possible solution is the Lefschetz thimble approach. It requires a deformation of the original integration domain into a manifold embedded in complex space. For properly chosen integration manifolds ("thimbles") the sign problem is drastically alleviated. For some bosonic and fermionic models this approach has been shown to work. Here we apply the thimble disretization to $(0+1)$-dimensional QCD with standard staggerd quarks and disscuss issues that may arrise in higher dimensions.
\end{abstract}

34th annual International Symposium on Lattice Field Theory

24-30 July 2016

University of Southampton, UK

\footnotetext{
${ }^{*}$ Speaker.
} 


\section{Introduction}

Lattice QCD, which has proven to be extremely successful at zero baryon number density, is hampered by the infamous sign problem once a nonzero baryon chemical potential $\left(\mu_{B}\right)$ is introduced. The reason lies in the complex phase that is acquired by the fermion determinant at $\mu_{B}>0$. The fermion determinant, which is obtained after integrating out the Grassmann valued fermion fields from the QCD partition function is part of the weight in lattice QCD calculations. It is subject to importance sampling during a Monte Carlo integration of the grand canonical partition function. On a Euclidean hyper-cubic lattice one finds

$$
Z\left(T, V, \mu_{B}\right)=\int \prod_{x, v} \mathrm{~d} U_{x, v} \operatorname{det}\left[\mathrm{M}\left(\mathrm{U}_{\mathrm{x}, v}, \mu_{\mathrm{B}}\right)\right] e^{-S_{G}\left(U_{x, v}\right)} \equiv \int \prod_{x, v} \mathrm{~d} U_{x, v} e^{-S_{\text {eff }}\left(U_{x, v}, \mu_{B}\right)},
$$

where $S_{G}$ denotes the Euclidean gauge action, $x$ labels the space-time points and $v$ the space-time directions. $U_{x, v}$ are the $\mathrm{SU}(3)$-valued link variables, that are associated with the gauge fields in the continuum. The integral has dimension $n=8 \times 4 \times V$, where $V$ is the number of lattice points. As soon as the determinant is not a real and positive function anymore, standard MC methods break down. The so-called "sign problem" arises from the fluctuating sign of the determinant that leads to extreme cancellations and an exponentially suppressed signal-to-noise ratio.

In the past, many attempts have been made to circumvent this problem, including reweighting [1], Taylor expansion in the chemical potential [2, 3], analytic continuation from purely imaginary chemical potentials $[4,5]$, canonical partition functions [6, 7] and strong coupling methods $[8,9]$. However, they all have certain limitations. Two of the most recent strategies are based on a complexification of the integration variables $[10,11]$. In case of the link variables, one exploits Gell-Mann's representation $U_{x, \mu}=\exp \left\{-\frac{i}{2} \sum_{a} \omega_{x, v}^{a} \lambda^{a}\right\}$, where $\lambda_{a}$ denote the Gell-Mann matrices. The complexification is performed by allowing the real parameters $\omega_{x, v}^{a}$ to become generally complex, i.e. the link variables become elements of $\operatorname{SL}(3, \mathbb{C})$. A new idea recently put forward is to deform the original domain of integration from the real hyperplane to some submanifold of the complex space. Picard-Lefschetz theory tells us that associated with each complex saddle point $\sigma$ of the effective action $S_{\text {eff }}$ as introduced in Eq. (1.1), there exists a manifold $\mathbb{J}_{\sigma}$ ("thimble") such that the integral $Z$ can be written as a linear combination of Integrals over the thimbles,

$$
Z=\sum_{\sigma} n_{\sigma} I_{\sigma}, \quad \text { with } \quad I_{\sigma}=\int_{\mathbb{J}_{\sigma}} \prod_{x, v} \mathrm{~d} U_{x, v} e^{-S_{\text {eff }}\left(U_{x, v}, \mu_{B}\right)} \quad \text { and } \quad n_{\sigma} \in \mathbb{Z} .
$$

The thimble manifolds exhibit the appealing feature that the imaginary part of $S_{\text {eff }}$ is constant for all points on the thimble, the integrals $I_{\sigma}$ are thus non oscillating and can thus be performed numerically. Motivated by Witten's work $[12,13]$, this approach has been recently applied to various bosonic and fermionic models, for a recent review see [14].

\section{QCD in $(0+1)$-dimensions with diagonal Polyakov loops}

It is well known that the QCD partition function in $(0+1)$-dim. simplifies drastically. Due to the low dimensionality there is no gauge action and we are left with the fermion determinant only, 
which can be expressed as a determinant over a reduced matrix $M^{\text {red }}$ that depends on the Polyakov loop $(P)$ and anti-Polyakov loop $\left(P^{-1}\right)$. The partition function can thus be expressed as [15]

$$
Z^{\left(N_{f}\right)}=\int \mathrm{d} P \operatorname{det}^{N_{f}}\left[M^{\text {red }}\right], \quad \text { with } \quad M^{\text {red }}=2 \cosh \left(\mu_{c} / T\right) \mathbf{1}+e^{\mu / T} P+e^{-\mu / T} P^{-1},
$$

where $\mathrm{d} P$ is the Haar measure over the gauge group $\mathrm{SU}(3)$. Here $\mu_{c}$ is the critical chemical potential, given in terms of the quark mass $(m)$ as

$$
a \mu_{c}=\operatorname{arcsinh}(a m),
$$

with $a$ being the lattice constant. In the following we will restrict ourselfs to the one flavor partition function $\left(N_{f}=1\right)$.

Before we start dealing with $\operatorname{SL}(3, \mathbb{C})$ valued integration variables, we observe that in this $(0+1)$-dim. case the Polyakov loop can be diagonalized. On the price of introducing an additional term, the Jacobian $\left(J_{H}\right)$, we can parametrize the Polyakov loop in terms of 2 phases $\theta_{1}$ and $\theta_{2}$ as

$$
P=\operatorname{diag}\left(e^{i \theta_{1}}, e^{i \theta_{2}}, e^{-i\left(\theta_{1}+\theta_{2}\right)}\right) .
$$

The Jaccobian is therefore given as

$$
J_{H}\left(\theta_{1}, \theta_{2}\right)=\frac{8}{3 \pi^{2}} \sin ^{2}\left(\frac{\theta_{1}-\theta_{2}}{2}\right) \sin ^{2}\left(\frac{2 \theta_{1}+\theta_{2}}{2}\right) \sin ^{2}\left(\frac{\theta_{1}+2 \theta_{2}}{2}\right) .
$$

As long as we are interested in the partition function itself, or observables that are invariant under transformations $P \rightarrow P^{\prime}=U P U^{-1}$, it suffices to generate diagonal Polyakov loops and the partition function (2.1) can be written as integral over the phases $\theta_{1}$ and $\theta_{2}$ as

$$
Z=\int \mathrm{d} \theta_{1} \mathrm{~d} \theta_{2} e^{-S_{\text {eff }}\left(\theta_{1}, \theta_{2}\right)}
$$

where we define the effective action as

$$
\begin{aligned}
S_{\text {eff }} & =-\ln J\left(\theta_{1}, \theta_{2}\right)-\ln \left\{2 \cosh \left(\hat{\mu}_{c}\right)+2 \cosh \left(\hat{\mu}+i \theta_{1}\right)\right\} \\
& -\ln \left\{2 \cosh \left(\hat{\mu}_{c}\right)+2 \cosh \left(\hat{\mu}+i \theta_{2}\right)\right\}-\ln \left\{2 \cosh \left(\hat{\mu}_{c}\right)+2 \cosh \left(\hat{\mu}-i\left[\theta_{1}+\theta_{2}\right]\right)\right\} .
\end{aligned}
$$

Here we denote $\hat{\mu}=\mu / T$. At zero $\mu$, this effective action diverges on the grey lines shown in Fig. 1 which naturally divide the phase space into separated regions. Inside of each region we find a single minimum of the effective action, its location is shown in Fig. 1 (left) as blue dot. The minima become saddle points of $\operatorname{Re}\left[S_{\text {eff }}\right]$ in the complexified space $\left(\operatorname{Re}\left[\theta_{1}\right], \operatorname{Re}\left[\theta_{2}\right], \operatorname{Im}\left[\theta_{1}\right], \operatorname{Im}\left[\theta_{2}\right]\right)$ of real-dimension 4 . The tangent space of each stable thimble at the critical points is spanned by the Takagi vectors of the Hessian matrix $\frac{\partial}{\partial \theta_{i}} \frac{\partial}{\partial \theta_{j}} S_{\text {eff }}$. Not surprisingly, at $\mu=0$ each tangent space as well as each thimble itself is real, and can be identified with each other. The thimbles just coincide with the triangular regions shown in Fig. 1 (left). Due to the symmetries of the reduced Haar measure it suffices to integrate only over one of the thimbles, i.e. each thimble gives the same contribution to the partition function and they correspond to the Weyl chambers of the reduced Haar measure.

At $\mu \neq 0$, we observe that the saddle points drift into complex space but come back to the real plane at very large $\mu$, as indicated in the middle and right panel of Fig. 1. Here the thimbles as 

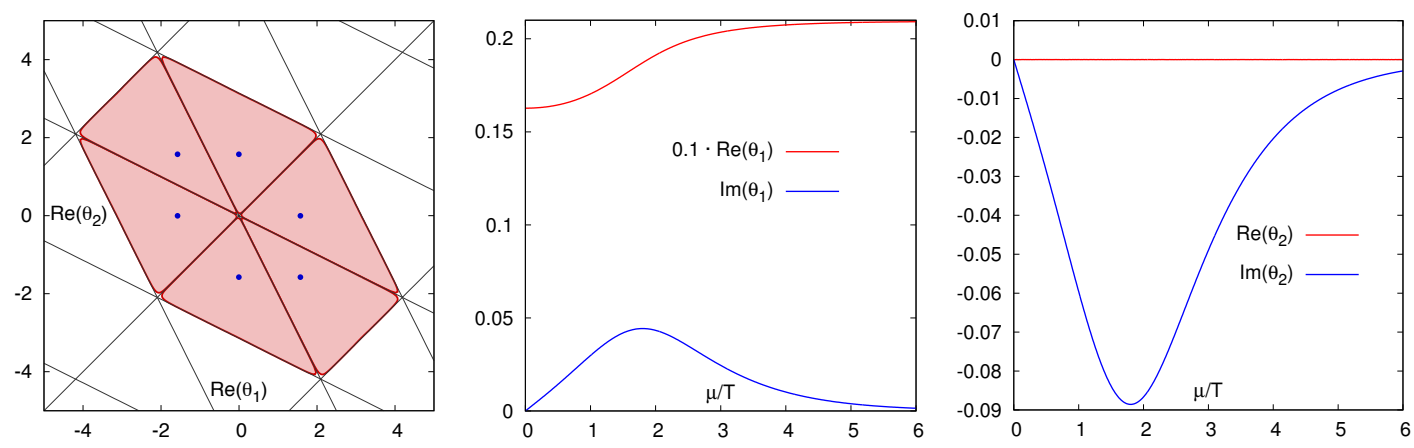

Figure 1: The Weyl chambers of the reduced Haar measure (left). For the first six chambers the saddle points at $\mu=0$ are indicated as dots. The dependence of the coordinates of the saddle points on the chemical potential are shown in the middle and right panel.

well as their tangent spaces at the saddle points $\left(\mathrm{T}_{\sigma} \mathbb{J}\right)$ become complex. The thimbles are now also curved manifolds which can not be identified with their tangent spaces.

To sample the thimble integrals (1.2) numerically, several algorithm have been proposed [11, $16,17,18,19]$. We will apply here the contraction algorithm proposed in [19]. It relies on the fact, that the thimble $\mathbb{J}$ can be generated from an $\varepsilon$-environment $U_{\varepsilon} \subset \mathrm{T}_{\sigma} \mathbb{J}$ around the saddle point $\sigma$ by making use of the flow map $F_{\tau}(\theta)$ of the initial value problem defined by the steepest ascent (SA) equation

$$
\frac{\mathrm{d}}{\mathrm{d} t} \theta=\left(\frac{\mathrm{d} S_{\mathrm{eff}}}{\mathrm{d} \theta}\right)^{*}, \quad \theta\left(t_{0}\right) \in U_{\varepsilon}
$$

Here $\theta$ denotes a vector of complex-dimension 2. For a fixed flow time $\tau>t_{0}, F_{\tau}(\theta): \mathbb{C}^{2} \rightarrow \mathbb{C}^{2}$ defines a map and in particular we have $F_{\tau}\left(U_{\varepsilon}\right) \simeq \mathbb{J}$ if $\varepsilon$ is sufficiently small. Note that we can not take the limit $\varepsilon \rightarrow 0$, as the image of $F_{\tau}$ will then be reduced to a single static point, the critical point $\sigma$. Note also that integrating the SA equation is numerically stable, thus for large flow times $\tau$, the flow will be pushed towards the stable thimble $\mathbb{J}$. The idea of the contraction algorithm is to integrate the thimble by sampling $\mathrm{T}_{\sigma} \mathbb{J}$, e.g. with a random-walk Metropolis algorithm. The algorithm is depicted graphically in Fig. 2. Mathematically, this procedure corresponds to a variable transformation of the integral. Thus, we have to take into account an additional Jacobian $J_{F}$, which we obtain by flowing a complete set of orthonormal basis vectors of the tangent space from the sampling point to the thimble point and taking the determinant to the linear map they define. It is clearly visible from Fig. 2 (right) that an anisotropic proposal probability is advisable, as the Takagi values of the Hessian are rather different.

The contraction algorithm can be used to calculate one-thimble expectation values of thermodynamic observables $O$, defined as

$$
\langle O\rangle_{\sigma}=\frac{1}{Z_{\sigma}} \int_{\mathbb{J}_{\sigma}} \mathrm{d} \theta_{1} \mathrm{~d} \theta_{2} O e^{-S_{\text {eff }}\left(\theta_{1}, \theta_{2}\right)}, \quad \text { with } \quad Z_{\sigma}=\int_{\mathbb{J}_{\sigma}} \mathrm{d} \theta_{1} \mathrm{~d} \theta_{2} e^{-S_{\text {eff }}\left(\theta_{1}, \theta_{2}\right)} .
$$

We stress again, that the integral over a single thimble is completely free of any sign problem stemming from the fermion determinant. There is, however, a much reduced residual sign problem, 

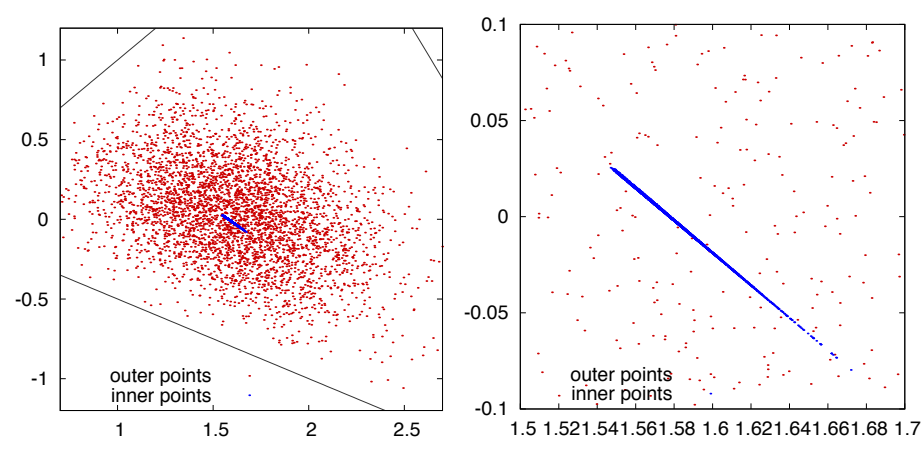

Figure 2: Right: Graphical represantation of the contraction map $F_{\tau}^{-1}(\theta)$ for $\mu=0$ and $\tau=0.7$. Sample points on the tangent space of the thimble are depicted in blue, while the red points indicate their corresponding images on the thimble. Left: The same but zoomed in towards the critical piont.

which due to the fact that the integration manifold is curved and is here taken into account through the complex valued Jacobian $J_{F}$.

It was pointed out in Ref. [20] that for short flow times $\tau$, the image $F_{\tau}\left(U_{\varepsilon}\right)$ does indeed come close to several thimbles. This way, the contraction algorithm for small $\tau$, can be used to obtain expectation values according to the correct partition function as defined in Eq. (1.1). This version of the contraction algorithm will be call Maryland algorithm in the following. Although the sign problem now comes back into the game, as the phase of the determinant is not constant on the considered manifold $F_{\tau}\left(U_{\varepsilon}\right)$, we find that after reweighting with the phase, the Maryland algorithm yields indeed correct expectation values for $(0+1)$-dimensional QCD.

\section{QCD in $(0+1)$-dimensions with general Polyakov loops}

We now come back to $\mathrm{SU}(3)$-valued variables and parameterize the Polyakov loop as $P=$ $\exp \left(-\frac{i}{2} \sum_{a} \omega_{a} \lambda_{a}\right)$. The number of independent variables is thus increased from 2 to 8 , leading to 16 degrees of freedom in the complexified theory. Without the Jacobian of the reduced Haar measure we find already at $\mu=0$ (up to periodicity) three inequivalent thimbles at $P=\mathbf{1}, e^{i 2 \pi / 3} \mathbf{1}$ and $e^{-i 2 \pi / 3} \mathbf{1}$, in correspondence with the holonomy sectors of the Polyakov loop under generalized gauge transformations. This structure remains valid for $\mu \neq 0$. The relative weight between the thimbles varies with $\mu$, whereas the critical points itself appear to be $\mu$-independent. In Fig. 3(left) we show the action of as function of the parameter $\operatorname{Re}\left[\omega_{8}\right]$ for $\mu=\mu_{c}$, which features the three critical points as local minima. When the thimbles are projected to the same space as shown in Fig. 1 (left), the plane spanned by the angle variables $\operatorname{Re}\left[\theta_{1}\right]$ and $\operatorname{Re}\left[\theta_{2}\right]$, which are now given as $\theta_{1}=-\omega_{3} / 2-\omega_{8} /(2 \sqrt{3})$ and $\theta_{2}=\omega_{3} / 2-\omega_{8} /(2 \sqrt{3})$, we find that they are bounded by the (projected) lines shown in Fig. 3 (middle).

As usual the tangent space of the thimbles at the critical points are spanned by the Takagi vectors of the Hessian, now given as

$$
H_{k l}=\frac{\partial^{2} S}{\partial \omega_{k} \partial \omega_{l}}=\operatorname{Tr}\left[M^{-1} \frac{\partial M}{\partial \omega_{l}} M^{-1} \frac{\partial M}{\partial \omega_{k}}-M^{-1} \frac{\partial^{2} M}{\partial \omega_{k} \partial \omega_{l}}\right]
$$



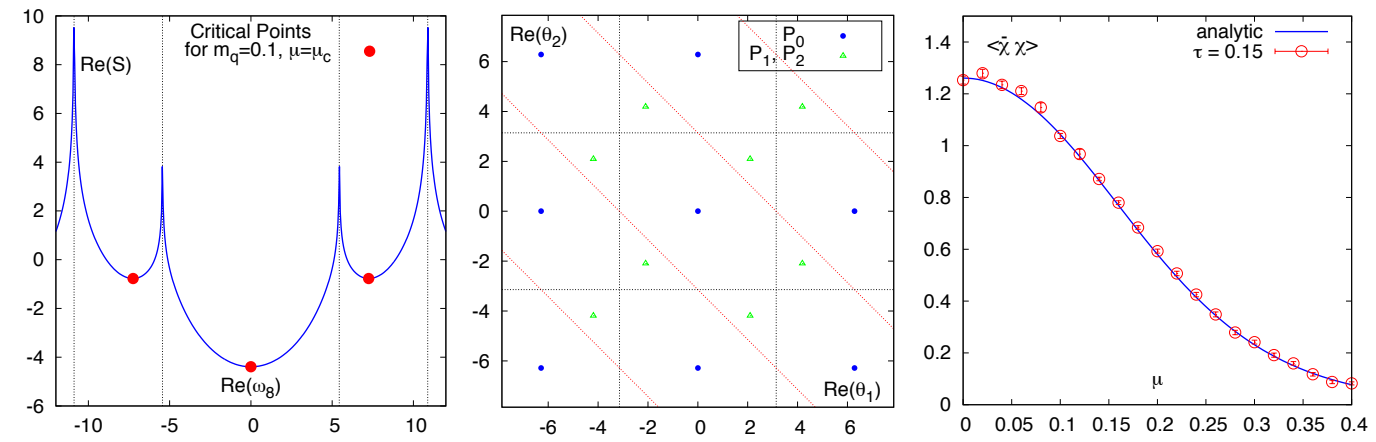

Figure 3: Left: Action as function of the parameter $\operatorname{Re}\left[\omega_{8}\right]$ for $\mu=\mu_{c}$ and am $=0.1$. Middle: Projection of critical points and thimbles onto the $\left(\theta_{1}, \theta_{2}\right)$-plane, as defined in the text. Right: Chiral condensate in $(0+1)$-dimensional QCD for $a m=0.1$ and $T=1 / 4 a$ as a function of $a \mu$. The results have been obtained with the contraction algorithm at flow time $\tau=0.15$. Also shown is the exact analytic result.

We find that at the critical points the Hessian takes the form $H=\gamma \mathbf{1}$, with a real valued $\gamma$ for the main thimble $\mathbb{J}_{0},(P=\mathbf{1})$, and a general complex $\gamma$ for the two others. As a consequence the tangent space at the three critical points do not coincide but are rotated with respect to each other. We believe that this fact renders the Maryland algorithm rather ineffective. Nevertheless, we find that it gives the correct results as shown in Fig. 3 (right) on the example of the chiral condensate.

We note that a more effective algorithm for this simple case of $(0+1)$-dimensional might be obtained by following the procedure as proposed in Ref. [21]. If the intersection $n_{\sigma}$ numbers are known, which is true in our case where all are equal to one, we can pick a thimble $\mathbb{J}_{\sigma}$ with probability $\frac{n_{\sigma}}{\sum n_{\sigma}}$, perform an update there and count how often the we accept to jump to a different thimble. This shall give the correct weight $c_{k}$ for the expectation value $\langle O\rangle_{\sigma}$, according to

$$
\langle O\rangle=\frac{\sum_{\sigma} n_{\sigma} e^{-i S_{I}\left(P_{\sigma}\right)} \int_{\mathbb{J}_{\sigma}} \mathrm{d} P O(P) e^{-S_{R}(P)}}{\sum_{\sigma} n_{\sigma} e^{-i S_{I}\left(P_{\sigma}\right)} Z_{\sigma}}=\sum_{\sigma} c_{\sigma}\langle O\rangle_{\sigma} .
$$

\section{Outlook: QCD in $(1+1)$-dimensions}

Now we have set the stage for $(1+1)$-dimensional QCD, which is work in progress. It shall be very interesting to see if the three basic thimbles remain, or if the thimble structure gets considerably complicated. Unfortunately, a reduced definition of the fermion matrix, given only in terms of Polyakov loops is no longer available. It will be desirable to work in temporal gauge (or any other fixed gauge), which reduces the number of integration variables, and avoids complications due to gauge orbits. Nevertheless, the computation of the Hessian, Eq. (3.1), will become numerically much more involved. Also, for the calculation of the action, the residual phase [22], or similarly the Jacobian in case of the contraction algorithm [23], one eventually needs to find a stochastic approximation.

\section{Aknowledgements}

We whish to thank W. Unger and A. Lindemeier for helpfull discussions and comments. 


\section{References}

[1] Z. Fodor and S. D. Katz, Phys. Lett. B 534 (2002) 87 [hep-lat/0104001].

[2] C. R. Allton, S. Ejiri, S. J. Hands, O. Kaczmarek, F. Karsch, E. Laermann, C. Schmidt and L. Scorzato, Phys. Rev. D 66 (2002) 074507 [hep-lat/0204010].

[3] R. V. Gavai and S. Gupta, Phys. Rev. D 68 (2003) 034506 [hep-lat/0303013].

[4] P. de Forcrand and O. Philipsen, Nucl. Phys. B 642 (2002) 290 [hep-lat/0205016].

[5] M. D'Elia and M. P. Lombardo, Phys. Rev. D 67 (2003) 014505 [hep-lat/0209146].

[6] A. Alexandru, M. Faber, I. Horvath and K. F. Liu, Phys. Rev. D 72 (2005) 114513 [hep-lat/0507020].

[7] S. Kratochvila and P. de Forcrand, PoS LAT 2005 (2006) 167 [hep-lat/0509143].

[8] T. Kloiber and C. Gattringer, PoS LATTICE 2013 (2014) 206 [arXiv:1310.8535 [hep-lat]].

[9] P. de Forcrand, J. Langelage, O. Philipsen and W. Unger, Phys. Rev. Lett. 113 (2014) no.15, 152002 [arXiv:1406.4397 [hep-lat]].

[10] D. Sexty, Phys. Lett. B 729 (2014) 108 [arXiv:1307.7748 [hep-lat]].

[11] M. Cristoforetti et al. [AuroraScience Collaboration], Phys. Rev. D 86 (2012) 074506 [arXiv:1205.3996 [hep-lat]].

[12] E. Witten, AMS/IP Stud. Adv. Math. 50 (2011) 347 [arXiv:1001.2933 [hep-th]].

[13] E. Witten, arXiv:1009.6032 [hep-th].

[14] L. Scorzato, PoS LATTICE 2015 (2016) 016 [arXiv:1512.08039 [hep-lat]].

[15] N. Bilic and K. Demeterfi, Phys. Lett. B 212 (1988) 83.

[16] H. Fujii, D. Honda, M. Kato, Y. Kikukawa, S. Komatsu and T. Sano, JHEP 1310 (2013) 147 [arXiv:1309.4371 [hep-lat]].

[17] A. Mukherjee and M. Cristoforetti, Phys. Rev. B 90 (2014) no.3, 035134 [arXiv:1403.5680 [cond-mat.str-el]].

[18] F. Di Renzo and G. Eruzzi, Phys. Rev. D 92 (2015) no.8, 085030 [arXiv:1507.03858 [hep-lat]].

[19] A. Alexandru, G. Basar and P. Bedaque, Phys. Rev. D 93 (2016) no.1, 014504 [arXiv:1510.03258 [hep-lat]].

[20] A. Alexandru, G. Basar, P. F. Bedaque, G. W. Ridgway and N. C. Warrington, JHEP 1605 (2016) 053 [arXiv:1512.08764 [hep-lat]].

[21] A. Mukherjee, M. Cristoforetti and L. Scorzato, Phys. Rev. D 88 (2013) no.5, 051502 [arXiv:1308.0233 [physics.comp-ph]].

[22] M. Cristoforetti, F. Di Renzo, G. Eruzzi, A. Mukherjee, C. Schmidt, L. Scorzato and C. Torrero, Phys. Rev. D 89 (2014) no.11, 114505 [arXiv:1403.5637 [hep-lat]].

[23] A. Alexandru, G. Basar, P. F. Bedaque, G. W. Ridgway and N. C. Warrington, arXiv:1604.00956 [hep-lat]. 